\title{
GLOBAL OPTIMIZATION METHOD FOR SOLVING MATHEMATICAL PROGRAMS WITH LINEAR COMPLEMENTARITY CONSTRAINTS
}

\author{
N.V. THOAI, Y. YAMAMOTO, AND A. YOSHISE
}

\begin{abstract}
We propose a method for finding a global optimal solution of programs with linear complementarity constraints. The program arises for instance from the bilevel programs. The main idea of the method is to generate a sequence of points either ending at a global optimal solution within a finite number of iterations or converging to a global optimal solution. The construction of such a sequence is based on the techniques such as branch and bound technique, which are used successfully in global optimization. Some results on a numerical test of the algorithm are reported.
\end{abstract}

\section{INTRODUCTION}

The mathematical programming problem to be considered in this article is characterized by the constraints described as a linear complementarity problem. This is a special case of the mathematical program with equilibrium constraints, and is a typical multiextremal global optimization problem in which local optimal solutions are in general different from global optimal solutions.

In [6] and references therein, a comprehensive study of mathematical programs with equilibrium constraints is presented including applications, theoretical results of exact penalty formulations, optimality conditions, and iterative algorithms. A sequential quadratic programming algorithm is proposed in [2]. An extreme point algorithm for computing a local optimal solution is presented recently in $[12]$. In $[9,10]$ some stability properties of a special class of programs with linear complementarity constraints are discussed and a cutting plane algorithm for computing an approximate optimal solution is proposed.

The purpose of the present article is to propose a method for finding a global optimal solution of programs with linear complementarity constraints. The main idea of this method is to generate a sequences of points either ending at a global optimal solution or converging to a global optimal solution of the problem under consideration. The construction of such a sequence is based on some techniques which are used successfully in global optimization. As we will see below, the problem considered in this paper belongs to a special class of MPECs (mathematical programs with equilibrium constraints) having the mixed $P_{0}$ property (cf. p.227 in [6]). This implies that most of the algorithms described in [6] can be applied to the problems. In contrast to those algorithms, our algorithms a remarkable feature that it only employs linear programs as its subproblems, by virtue of the special structure of the problem.

The article is organized as follows. The next section contains the exact formulation of the mathematical programming problem with linear complementarity constraints, and its equivalent form. Section 3 presents some relaxation forms of the problem, which are used to establish the

Date: May 7, 2002; revised May 13, 2002.

Key words and phrases. Programs with linear complementarity constraints, Equilibrium constraints, Bilevel programming, Global optimization. 
algorithm in Section 4. An illustrative example and some results of preliminary computational experiments are reported in Section 5.

\section{Problem Formulation}

We are concerned with the following mathematical programming problem:

$$
\begin{array}{ll}
\min & f(z, \lambda) \\
\text { s.t. } & z \in Z \\
& (z, \lambda) \in \Omega \\
& B z+b \leq 0 \\
& \lambda^{\top}(B z+b)=0 \\
& \lambda \geq 0,
\end{array}
$$

where $Z$ and $\Omega$ are convex closed subsets of $\mathbb{R}^{n}$ and $\mathbb{R}^{n+p}$, respectively, $f: \mathbb{R}^{n+p} \rightarrow \mathbb{R}$ is a continuous function, $B \in \mathbb{R}^{p \times n}$ is a given matrix, and $b \in \mathbb{R}^{p}$ is a given vector. We assume throughout the paper that $Z$ is bounded.

Problem (PC) contains as constraints a system of the form

$$
\begin{aligned}
& B z+b \leq 0 \\
& \lambda^{\top}(B z+b)=0 \\
& \lambda \geq 0,
\end{aligned}
$$

which is well known as the linear complementarity problem. The theory and solution methods for the linear complementarity problem can be found, e.g., in [1] and [5]. Characterized by the linear complementarity (C), Problem (PC) is frequently called the mathematical program with linear complementarity constraints. ${ }^{1}$ Even for the case where $Z$ is a polyhedral set and $f$ is a linear function, Problem (PC) is a typical multiextremal global optimization problem, where local optimal solutions are in general different from global optimal solutions.

Let $w=-B z-b$. Then there exists a polytope (i.e., bounded polyhedron), say $W$, in $\mathbb{R}_{+}^{p}$, whose construction shall be discussed later in Section 4, so that Problem (PC) can be rewritten as

\footnotetext{
${ }^{1} \mathrm{~A}$ special case of the MPEC given below is dealt with in [2]:

$$
\begin{array}{ll}
\min & f(z, \lambda) \\
\text { s.t. } & z \in Z \\
& (z, \lambda) \in \Omega \\
& B z+C \lambda+b \leq 0 \\
& \lambda^{\top}(B z+C \lambda+b)=0
\end{array}
$$$$
\lambda \geq 0 \text {. }
$$

When the matrix $C$ is a $P_{0}$ matrix, the problem is said to have the $P_{0}$ property. Since $C$ of Problem (PC) is a zero matrix, which is obviously a $P_{0}$ matrix, Problem (PC) has the $P_{0}$ property.
} 


$$
\begin{array}{ll}
\min & f(z, \lambda) \\
\text { s.t. } & z \in Z \\
& (z, \lambda) \in \Omega \\
& B z+w+b=0 \\
& \lambda^{\top} w=0 \\
& \lambda \geq 0, \\
& w \in W \subset \mathbb{R}_{+}^{p} .
\end{array}
$$

Clearly, Problems $(\mathrm{PC})$ and $(\mathrm{P})$ are equivalent in the sense that

(i) If $\left(z^{*}, \lambda^{*}\right)$ is an optimal solution of (PC), then $\left(z^{*}, \lambda^{*}, w^{*}\right)$ with $w^{*}=-B z^{*}-b$ is an optimal solution of $(\mathrm{P})$, and

(ii) If $\left(z^{*}, \lambda^{*}, w^{*}\right)$ is an optimal solution of (P), then $\left(z^{*}, \lambda^{*}\right)$ is an optimal solution of (PC).

Using the equivalent formulation (P), we develop a method for globally solving Problem (PC). The main idea can be sketched out as follows. Let $\bar{\Gamma}$ be the feasible set of Problem (P), and let

$$
\Gamma=\{(z, \lambda, w): z \in Z,(z, \lambda) \in \Omega, B z+w+b=0, \lambda \geq 0, w \in W\} .
$$

The algorithm generates a sequence $\left\{\left(z^{k}, \lambda^{k}, w^{k}\right)\right\}_{k=1,2, \ldots} \subset \Gamma$ having the property that, for each $k$, $f\left(z^{k}, \lambda^{k}\right)$ provides a lower bound of the optimal value of Problem (P). Such a sequence either ends at some iteration $k$ yielding an optimal solution or converges to an optimal solution of Problem $(\mathrm{P})$.

\section{Relaxation Problems}

Letting $S$ be any polytope contained in $W$ with the vertex set $V(S)=\left\{s^{1}, s^{2}, \cdots, s^{\pi}\right\}$, we consider the problem $(\mathrm{P}(\mathrm{S}))$ which is obtained from Problem $(\mathrm{P})$ by replacing $W$ by $S$ :

$$
\begin{array}{ll}
\min & f(z, \lambda) \\
\text { s.t. } \quad & z \in Z \\
& (z, \lambda) \in \Omega \\
& B z+w+b=0 \\
& \lambda^{\top} w=0 \\
& \lambda \geq 0, \\
& w \in S .
\end{array}
$$

Based on Problem $(\mathrm{P}(\mathrm{S}))$ we define the following programming problem in variables $\left(z, \lambda^{1}, \cdots, \lambda^{\pi}, \beta\right)$ with $z \in \mathbb{R}^{n}, \lambda^{i} \in \mathbb{R}^{p}(i=1, \cdots, \pi)$, and $\beta \in \mathbb{R}^{\pi}$ :

$$
\begin{array}{ll}
\min & f\left(z, \sum_{i=1}^{\pi} \lambda^{i}\right) \\
\mathrm{s.t.} & z \in Z \\
& \left(z, \sum_{i=1}^{\pi} \lambda^{i}\right) \in \Omega \\
& B z+\sum_{i=1}^{\pi} \beta_{i} s^{i}+b=0 \\
& \sum_{i=1}^{\pi} \beta_{i}=1 \\
& \sum_{i=1}^{\pi}\left(s^{i}\right)^{\top} \lambda^{i}=0 \\
& \lambda^{i} \geq 0(i=1, \cdots, \pi) \\
& \beta \geq 0
\end{array}
$$


The main distinction between Problem $\left(\mathrm{P}_{1}(\mathrm{~S})\right)$ and Problem $(\mathrm{P}(\mathrm{S}))$ is the introduction of the variables $\lambda^{1}, \cdots, \lambda^{\pi}$ and $\beta$ to represent $\lambda$ and $w$, respectively. This relaxes the nonlinear constraint $\lambda^{\top} w=0$ in Problem (P) to the linear constraint $\sum_{i=1}^{\pi}\left(s^{i}\right)^{\top} \lambda^{i}=0$ in Problem $\left(\mathrm{P}_{1}(\mathrm{~S})\right)$, which will be seen in the following proposition. This relaxation technique is used in [3] for the minimum maximal flow problem.

Theorem 3.1. Let $f(S)$ and $\mu_{1}(S)$ denote the optimal values of Problems $(P(S))$ and $\left(P_{1}(S)\right)$, respectively, and let $\left(z(S), \lambda^{1}(S), \cdots, \lambda^{\pi}(S), \beta(S)\right)$ be an optimal solution of Problem $\left(P_{1}(S)\right)$. Then

(i) $\mu_{1}(S) \leq f(S)$, i.e. Problem $\left(P_{1}(S)\right)$ is a relaxation of Problem $(P(S))$.

(ii) If $\left(\sum_{i=1}^{\pi} \beta_{i}(S) s^{i}\right)^{\top}\left(\sum_{i=1}^{\pi} \lambda^{i}(S)\right)=0$, then $(z(S), \lambda(S), w(S))$ with $\lambda(S)=\sum_{i=1}^{\pi} \lambda^{i}(S)$ and $w(S)=\sum_{i=1}^{\pi} \beta_{i}(S) s^{i}$ is an optimal solution of Problem $(P(S))$.

Proof. ( $i$ ) It suffices to show that for each feasible solution $(z, \lambda, w)$ of Problem $(\mathrm{P}(\mathrm{S}))$ there exists a feasible solution $\left(z, \lambda^{1}, \cdots, \lambda^{\pi}, \beta\right)$ of Problem $\left(\mathrm{P}_{1}(\mathrm{~S})\right)$ such that $\sum_{i=1}^{\pi} \lambda^{i}=\lambda$. Note that each $w \in S$ is represented by

$$
w=\sum_{\ell=1}^{\pi} \beta_{i} s^{i}, \beta_{i} \geq 0(i=1, \cdots, \pi), \sum_{i=1}^{\pi} \beta_{i}=1 .
$$

Define

$$
\lambda^{i}=\beta_{i} \lambda \text { for } i=1, \cdots, \pi .
$$

Then clearly $\sum_{i=1}^{\pi} \lambda^{i}=\lambda$ and $\left(z, \lambda^{1}, \cdots, \lambda^{\pi}, \beta\right)$ satisfies the first four groups of constraints of Problem $\left(\mathrm{P}_{1}(\mathrm{~S})\right)$ as well as $\lambda^{i} \geq 0(i=1, \cdots, \pi)$ and $\beta \geq 0$. For the remaining constraint, from (3.1), we have

$$
\sum_{i=1}^{\pi}\left(s^{i}\right)^{\top} \lambda^{i}=\sum_{i=1}^{\pi} \beta_{i}\left(s^{i}\right)^{\top} \lambda=\lambda^{\top} w=0
$$

Thus, $\left(z, \lambda^{1}, \cdots, \lambda^{\pi}, \beta\right)$ is a feasible solution of Problem $\left(\mathrm{P}_{1}(\mathrm{~S})\right)$.

(ii) Let $\left(z(S), \lambda^{1}(S), \cdots, \lambda^{\pi}(S), \beta(S)\right)$ be an optimal solution of Problem $\left(\mathrm{P}_{1}(\mathrm{~S})\right)$, and let

$$
\lambda(S)=\sum_{i=1}^{\pi} \lambda^{i}(S), w(S)=\sum_{i=1}^{\pi} \beta_{i}(S) s^{i} .
$$

Then obviously, $(z(S), \lambda(S), w(S))$ satisfies all the constraints of Problem $(\mathrm{P}(\mathrm{S}))$ except for $\lambda(S)^{\top} w(S)=$ 0 . Thus, if

$$
\left(\sum_{i=1}^{\pi} \beta_{i}(S) s^{i}\right)^{\top}\left(\sum_{i=1}^{\pi} \lambda^{i}(S)\right)=w(S)^{\top} \lambda(S)=0
$$

$(z(S), \lambda(S), w(S))$ is a feasible solution of Problem $(\mathrm{P}(\mathrm{S}))$, and therefore, it follows from $(i)$ that $(z(S), \lambda(S), w(S))$ is an optimal solution of Problem $(\mathrm{P}(\mathrm{S}))$.

Next, we show that the optimal value of the relaxation problem has an useful monotonicity property.

Lemma 3.2. If two polytopes $S^{1}$ and $S^{2}$ contained in $W$ satisfy $S^{2} \subset S^{1}$, then

$$
\mu_{1}\left(S^{1}\right) \leq \mu_{1}\left(S^{2}\right) .
$$


Proof. Let $V\left(S^{1}\right)=\left\{s^{11}, \cdots, s^{1 \pi_{1}}\right\}$ and $V\left(S^{2}\right)=\left\{s^{21}, \cdots, s^{2 \pi_{2}}\right\}$ be the vertex sets of $S^{1}$ and $S^{2}$, respectively. We show that to each feasible solution $\left(z, \lambda^{21}, \cdots, \lambda^{2 \pi_{2}}, \beta^{2}\right)$ of Problem $\left(\mathrm{P}_{1}\left(S^{2}\right)\right)$ there exists a feasible solution $\left(z, \lambda^{11}, \cdots, \lambda^{1 \pi_{1}}, \beta^{1}\right)$ of Problem $\left(\mathrm{P}_{1}\left(S^{1}\right)\right)$ satisfying

$$
f\left(z, \sum_{i=1}^{\pi_{1}} \lambda^{1 i}\right)=f\left(z, \sum_{i=1}^{\pi_{2}} \lambda^{2 i}\right) .
$$

First, let $w=\sum_{i=1}^{\pi_{2}} \beta_{i}^{2} s^{2 i}$. Then $w \in S^{2} \subset S^{1}$. Therefore, there exists a vector $\beta^{1} \geq 0$ satisfying

$$
w=\sum_{i=1}^{\pi_{1}} \beta_{i}^{1} s^{1 i}, \sum_{i=1}^{\pi_{1}} \beta_{i}^{1}=1 .
$$

Next, Since $S^{2} \subset S^{1}$, to each vertex $s^{2 i}\left(i=1, \cdots, \pi_{2}\right)$ there exists a vector $\sigma^{i} \geq 0$ such that

$$
s^{2 i}=\sum_{j=1}^{\pi_{1}} \sigma_{j}^{i} s^{1 j}, \sum_{j=1}^{\pi_{1}} \sigma_{j}^{i}=1 .
$$

Using these vectors $\sigma^{i}\left(i=1, \cdots, \pi_{2}\right)$, we define vectors $\lambda^{1 i}\left(i=1, \cdots, \pi_{1}\right)$ by

$$
\lambda^{1 i}=\sum_{j=1}^{\pi_{2}} \sigma_{i}^{j} \lambda^{2 j}
$$

Then it follows that

$$
\sum_{i=1}^{\pi_{1}} \lambda^{1 i}=\sum_{i=1}^{\pi_{1}} \sum_{j=1}^{\pi_{2}} \sigma_{i}^{j} \lambda^{2 j}=\sum_{j=1}^{\pi_{2}} \sum_{i=1}^{\pi_{1}} \sigma_{i}^{j} \lambda^{2 j}=\sum_{j=1}^{\pi_{2}} \lambda^{2 j} \sum_{i=1}^{\pi_{1}} \sigma_{i}^{j}=\sum_{j=1}^{\pi_{2}} \lambda^{2 j},
$$

implying (3.2). It is straightforward to see that $\left(z, \lambda^{11}, \cdots, \lambda^{1 \pi_{1}}, \beta^{1}\right)$ is a feasible solution of Problem $\left(\mathrm{P}_{1}\left(S^{1}\right)\right)$. As an example, the equality

$$
\sum_{i=1}^{\pi_{1}}\left(s^{1 i}\right)^{\top} \lambda^{1 i}=0
$$

is proved from (3.3) and (3.4) as

$$
\sum_{i=1}^{\pi_{1}}\left(s^{1 i}\right)^{\top} \lambda^{1 i}=\sum_{i=1}^{\pi_{1}}\left(s^{1 i}\right)^{\top} \sum_{j=1}^{\pi_{2}} \sigma_{i}^{j} \lambda^{2 j}=\sum_{j=1}^{\pi_{2}}\left(\sum_{i=1}^{\pi_{1}} \sigma_{i}^{j} s^{1 i}\right)^{\top} \lambda^{2 j}=\sum_{j=1}^{\pi_{2}}\left(s^{2 j}\right)^{\top} \lambda^{2 j}=0 .
$$

The last equation follows from the feasibility of $\left(z, \lambda^{21}, \cdots, \lambda^{2 \pi}, \beta^{2}\right)$ to Problem $\left(\mathrm{P}_{1}\left(S^{2}\right)\right)$.

When the polytope $S \subset W$ is a rectangle given by $0 \leq \underline{s} \leq \bar{s}$ as

$$
S=\left\{w \in \mathbb{R}_{+}^{p}: \underline{s} \leq w \leq \bar{s}\right\}
$$

Problem $\left(\mathrm{P}_{2}(S)\right)$ below is also a relaxation of $(\mathrm{P}(S))$ with the same monotonicity property as $\left(\mathrm{P}_{1}(S)\right)$.

$$
\begin{array}{ll}
\min & f(z, \lambda) \\
\text { s.t. } \quad & z \in Z \\
& (z, \lambda) \in \Omega \\
& B z+w+b=0 \\
& \lambda^{\top} \underline{s}=0 \\
& \lambda \geq 0, \\
& w \in S \subset \mathbb{R}_{+}^{p},
\end{array}
$$


Theorem 3.3. Problem $\left(P_{2}(S)\right)$ is a relaxation of Problem $(P(S))$ in the sense that

(i) $\mu_{2}(S) \leq f(S)$, where $\mu_{2}(S)$ denotes the optimal value of Problem $\left(P_{2}(S)\right)$.

(ii) If $w(S)^{\top} \lambda(S)=0$, where $(z(S), \lambda(S), w(S))$ is an optimal solution of Problem $\left(P_{2}(S)\right)$, then $(z(S), \lambda(S), w(S))$ is an optimal solution of Problem $(P(S))$.

(iii) If $S^{1}, S^{2}$ are rectangles satisfying $S^{2} \subset S^{1} \subset W$, then $\mu_{2}\left(S^{1}\right) \leq \mu_{2}\left(S^{2}\right)$.

Remark 3.4. Note that for $u=\left(z, \lambda^{1}, \ldots, \lambda^{\pi}\right) \in \mathbb{R}^{n+\pi p}$ the vector $v=\left(z, \sum_{i=1}^{\pi} \lambda^{i}\right) \in \mathbb{R}^{n+p}$ is given by $v=L u$ where

$$
L=\left(\begin{array}{cccc}
I_{n} & 0 & 0 & O \\
0 & I_{p} & I_{p} & I_{p}
\end{array}\right)
$$

where $I_{k}$ is the identity matrix of dimension $k$. Let us define $G_{1}: \mathbb{R}^{n+\pi p} \rightarrow \mathbb{R}$ by $G_{1}(u)=G(L u)$ for a given function $G: \mathbb{R}^{n+p} \rightarrow \mathbb{R}$. If the function $G$ is convex on $\mathbb{R}^{n+p}$, we easily see that $G_{1}$ is also convex on $\mathbb{R}^{n+\pi p}$. $^{2}$

Assume that the convex closed set $\Omega$ of Problem $(P)$ is given by a convex vector function $G$ : $\mathbb{R}^{n+p} \rightarrow \mathbb{R}^{m}$, i.e., each component $g_{i}$ of $G$ is a convex function, as

$$
\Omega=\left\{(z, \lambda) \in \mathbb{R}^{n+p}: G(z, \lambda) \leq 0\right\}
$$

Then in Problem $\left(P_{1}(S)\right)$ the constraint $\left(z, \sum_{i=1}^{\pi} \lambda^{i}\right) \in \Omega$ is represented by $G_{1}\left(z, \lambda^{1}, \cdots, \lambda^{\pi}\right)=$ $G\left(z, \sum_{i=1}^{\pi} \lambda^{i}\right) \leq 0$. Clearly $G_{1}\left(z, \lambda^{1}, \cdots, \lambda^{\pi}\right)$ is also a convex vector function, so that in Problem $\left(P_{1}(S)\right)$, the set $\left\{\left(z, \lambda^{1}, \cdots, \lambda^{\pi}\right): G_{1}\left(z, \lambda^{1}, \cdots, \lambda^{\pi}\right) \leq 0\right\}$ remains a convex set. Thus, if the function $f(z, \lambda)$ is convex, then so is the function $f_{1}\left(z, \lambda^{1}, \cdots, \lambda^{\pi}\right)=f\left(z, \sum_{i=1}^{\pi} \lambda^{i}\right)$, and Problem $\left(P_{1}(S)\right)$ is a convex program. If the function $f(z, \lambda)$ is linear, and $Z$ and $\Omega$ are polyhedral sets, then $\left(P_{1}(S)\right)$ is an ordinary linear program.

\section{The Algorithm}

Based on the relaxation problems constructed in the previous section, we establish an algorithm for globally solving Problem (P).

For the sake of representation, to each optimal solution

$$
\left(z(S), \lambda^{1}(S), \cdots, \lambda^{\pi}(S), \beta(S)\right)
$$

of the relaxation problem $\left(\mathrm{P}_{1}(\mathrm{~S})\right)$, we assign a point $(z(S), \lambda(S), w(S))$ defined by

$$
\lambda(S)=\sum_{i=1}^{\pi} \lambda^{i}(S), w(S)=\sum_{i=1}^{\pi} \beta_{i}(S) s^{i},
$$

and will say that $(z(S), \lambda(S), w(S))$ is an optimal solution of Problem $\left(\mathrm{P}_{1}(\mathrm{~S})\right)$.

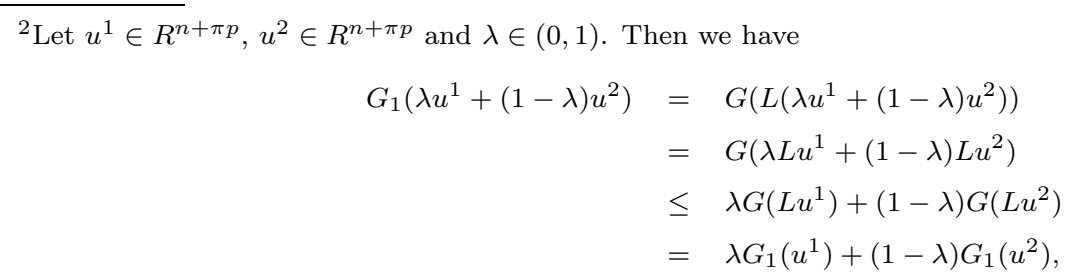

which implies that $G_{1}$ is also convex on $R^{n+\pi n}$. 
The main idea of the following algorithm is to construct a sequence of polytopes $\left\{S^{k}\right\}_{k=1,2, \ldots}$ and the corresponding sequence

$$
\left\{\left(z^{k}, \lambda^{k}, w^{k}\right)\right\}_{k=1,2, \ldots}=\left\{\left(z\left(S^{k}\right), \lambda\left(S^{k}\right), w\left(S^{k}\right)\right)\right\}_{k=1,2, \ldots}
$$

such that

(i) either $\left(z^{k}, \lambda^{k}, w^{k}\right)$ is a global optimal solution of Problem (P) for some $k$, or

(ii) each accumulation point of $\left\{\left(z^{k}, \lambda^{k}, w^{k}\right)\right\}_{k=1,2, \ldots}$ is a global optimal solution of Problem (P).

\subsection{Global Optimization Algorithm (GOA).}

$\langle\langle$ Initialization $\rangle\rangle$

Construct a simplex (or a rectangle) $W \subset \mathbb{R}_{+}^{p}$ containing at least a vector $w^{*}$ such that $w^{*}$ together with some $z^{*} \in \mathbb{R}^{n}$ and $\lambda^{*} \in \mathbb{R}_{+}^{p}$ forms an optimal solution $\left(z^{*}, \lambda^{*}, w^{*}\right)$ of Problem $(\mathrm{P})$. Set $S^{1}=W$. Solve the relaxation problem $\left(\mathrm{P}_{1}\left(\mathrm{~S}^{1}\right)\right)$ (or $\left(\mathrm{P}_{2}\left(\mathrm{~S}^{1}\right)\right)$ ), obtaining an optimal solution $\left(z^{1}, \lambda^{1}, w^{1}\right)=\left(z\left(S^{1}\right), \lambda\left(S^{1}\right), w\left(S^{1}\right)\right)$ and the optimal value $f\left(z^{1}, \lambda^{1}\right)$. Set $\mu^{1}=f\left(z^{1}, \lambda^{1}\right)$. If $\left(w^{1}\right)^{\top} \lambda^{1}=0$, then set $\gamma^{1}=f\left(z^{1}, \lambda^{1}\right)$ and $\left(\bar{z}^{1}, \bar{\lambda}^{1}, \bar{w}^{1}\right)=\left(z^{1}, \lambda^{1}, w^{1}\right)$. Otherwise, set $\gamma^{1}=+\infty$. Set $k=1$ and $\mathcal{R}_{1}=\left\{S^{1}\right\}$.

$\langle\langle$ Iteration $k\rangle\rangle$

Execute Steps (i) to (vi) below.

(i) If $\gamma^{k}=\mu^{k}$, then stop. $\left(\bar{z}^{k}, \bar{\lambda}^{k}, \bar{w}^{k}\right)$ is an optimal solution of Problem (P) with the optimal value $\gamma^{k}$.

(ii) If $\gamma^{k}>\mu^{k}$, then divide $S^{k}$ into $S_{1}^{k}, \ldots, S_{\nu}^{k}$ satisfying

$$
\bigcup_{j=1}^{\nu} S_{j}^{k}=S^{k} \text { and } \operatorname{int} S_{j}^{k} \cap \operatorname{int} S_{j^{\prime}}^{k}=\emptyset \text { for } j \neq j^{\prime}
$$

where $\operatorname{int} A$ denotes the interior of set $A$.

(iii) For each $j=1, \ldots, \nu$, solve Problem $\left(\mathrm{P}_{1}\left(\mathrm{~S}_{j}^{k}\right)\right)$ (or $\left(\mathrm{P}_{2}\left(\mathrm{~S}_{j}^{k}\right)\right.$ ), obtaining an optimal solution

$$
\left(z\left(S_{j}^{k}\right), \lambda\left(S_{j}^{k}\right), w\left(S_{j}^{k}\right)\right)
$$

and the optimal value $f\left(z\left(S_{j}^{k}\right), \lambda\left(S_{j}^{k}\right)\right)$. Set $\mu\left(S_{j}^{k}\right)=f\left(z\left(S_{j}^{k}\right), \lambda\left(S_{j}^{k}\right)\right)$.

If $w\left(S_{j}^{k}\right)^{\top} \lambda\left(S_{j}^{k}\right)=0$, then update

$$
\gamma^{k}=\min \left\{\gamma^{k}, f\left(z\left(S_{j}^{k}\right), \lambda\left(S_{j}^{k}\right)\right)\right\},
$$

and update, if necessary, the current best feasible solution $\left(\bar{z}^{k}, \bar{\lambda}^{k}, \bar{w}^{k}\right)$ according to $\gamma^{k}$.

(iv) Set

$$
\begin{aligned}
& \gamma^{k+1}=\gamma^{k}, \\
& \left(\bar{z}^{k+1}, \bar{\lambda}^{k+1}, \bar{w}^{k+1}\right)=\left(\bar{z}^{k}, \bar{\lambda}^{k}, \bar{w}^{k}\right), \\
& \mathcal{R}_{k+1}=\mathcal{R}_{k} \backslash\left\{S^{k}\right\} \bigcup\left\{S_{j}^{k}: j=1, \cdots, \nu\right\}, \\
& \mu^{k+1}=\min \left\{\mu(S): S \in \mathcal{R}_{k+1}\right\} .
\end{aligned}
$$

(v) Choose $S \in \mathcal{R}_{k+1}$ such that $\mu(S)=\mu^{k+1}$, and set $S^{k+1}=S$,

$$
\left(z^{k+1}, \lambda^{k+1}, w^{k+1}\right)=\left(z\left(S^{k+1}\right), \lambda\left(S^{k+1}\right), w\left(S^{k+1}\right)\right) .
$$

(vi) Go to iteration $k+1$. 
The above algorithm generates a sequence $\left\{\left(z^{k}, \lambda^{k}, w^{k}\right), \mu^{k}, \gamma^{k}\right\}_{k=1,2, \ldots}$ If an occasion should arise, it also generates a sequence $\left\{\left(\bar{z}^{k}, \bar{\lambda}^{k}, \bar{w}^{k}\right)\right\}_{k=1,2, \ldots}$. We show that $\left\{\mu^{k}\right\}_{k=1,2, \ldots}$ is a nondecreasing sequence of lower bounds of the optimal value of Problem (P). We also show that if the algorithm terminates at some iteration $k$ by the stopping criterion $\gamma^{k}=\mu^{k}$, then the point $\left(\bar{z}^{k}, \bar{\lambda}^{k}, \bar{w}^{k}\right)$ is an optimal solution.

Lemma 4.1. $\left\{\mu^{k}\right\}_{k=1,2, \ldots}$ is a nondecreasing sequence of lower bounds of the optimal value of $(P)$.

Proof. It is straightforward to show that $\mu^{k}$ is a lower bound of the optimal value of $(\mathrm{P})$. We only show the monotonicity. By the choice of $\mu^{k}$ in Step (iv) and $S^{k}$ in Step (v) we see that $\mu\left(S^{k}\right)=\mu^{k} \leq \mu(S)$ for any $S \in \mathcal{R}_{k}$. Since the sets $S_{1}^{k}, \ldots, S_{\nu}^{k}$ added to $\mathcal{R}_{k}$ to construct $\mathcal{R}_{k+1}$ are subsets of $S^{k}$, we obtain from Lemma 3.2 the inequality $\mu\left(S^{k}\right) \leq \mu\left(S_{j}^{k}\right)$ for $j=1,2, \ldots, \nu$. Therefore $\mu^{k} \leq \mu(S)$ for any $S \in \mathcal{R}_{k+1}$, implying the desired monotonicity $\mu^{k} \leq \mu^{k+1}$.

Lemma 4.2. When (GOA) terminates in Step (i), $\left(\bar{z}^{k}, \bar{\lambda}^{k}, \bar{w}^{k}\right)$ is an optimal solution of Problem $(P)$.

Proof. Note that $f\left(z^{k}, \lambda^{k}\right)=\gamma^{k}=\mu^{k}$ holds when the algorithm terminates, and $\mu^{k}$ is a lower bound of the optimal value of (P) by Lemma 4.1. These facts together with the feasibility of $\left(z^{k}, \lambda^{k}, w^{k}\right)$ yield the lemma.

Before establishing the convergence of the algorithm for the case where it does not terminate after finitely many iterations, we discuss some details of an implementation of this algorithm.

4.2. Construction of the first polytope. Since the set $Z$ is compact, there exist nonnegative numbers $\zeta_{j}, \xi_{j}(j=1, \cdots, p)$ defined by

$$
\begin{gathered}
\zeta_{j}=\max \left\{0, \min \left\{w_{j}: w=-B z-b, z \in Z,(z, \lambda) \in \Omega, \lambda \geq 0,\right.\right. \\
\left.\left.w_{i} \geq 0 \text { for } i \neq j\right\}\right\}, \\
\xi_{j}=\max \left\{w_{i}: w=-B z-b, z \in Z,(z, \lambda) \in \Omega, \lambda \geq 0,\right. \\
\left.w_{i} \geq \zeta_{i} \text { for } i=1,2, \ldots, p\right\} .
\end{gathered}
$$

If $\xi_{j}<0$ for some $j$, then it follows that Problem (PC) is infeasible, because the constraint $B z+b \leq 0$ cannot be satisfied for any $z$ with $z \in Z,(z, \lambda) \in \Omega, \lambda \geq 0$.

Assuming that $\xi_{j} \geq 0$ for all $j=1, \cdots, p$, we define further a number $\theta$ and a set $W$ by

$$
\begin{aligned}
& \theta=\max \left\{\sum_{i=1}^{p} w_{i}: w=-B z-b, z \in Z,(z, \lambda) \in \Omega, \lambda \geq 0, w \geq \zeta\right\}, \\
& W=\left\{w \in \mathbb{R}^{p}: w_{j} \geq \zeta_{j}(j=1, \cdots, p), \sum_{j=1}^{p} w_{j} \leq \theta\right\} .
\end{aligned}
$$

Then clearly, each optimal solution $\left(z^{*}, \lambda^{*}, w^{*}\right)$ of Problem $(\mathrm{P})$ satisfies the condition $w^{*} \in W$. Note that $W$ is a $p$-simplex having $p+1$ vertices $w^{1}, \cdots, w^{p+1}$, where

$$
w^{p+1}=\left(\zeta_{1}, \cdots, \zeta_{p}\right)
$$


and for each $j=1, \cdots, p$ the vertex $w^{j}$ is defined by

$$
\begin{aligned}
w_{j}^{j} & =\theta-\sum_{i=1}^{p} \zeta_{i}+\zeta_{j} \\
w_{i}^{j} & =\zeta_{i} \quad(i=1, \cdots, p ; i \neq j) .
\end{aligned}
$$

Alternate choice of the set $W$ is a rectangle defined by

$$
W=\left\{w \in \mathbb{R}^{p}: \zeta_{i} \leq w_{i} \leq \xi_{i}(i=1, \cdots, p)\right\}
$$

4.3. Simplicial Division. If $W$ is defined by (4.2), the following simplicial division should be used. Let $S$ be an $p$-simplex with vertex set $V(S)=\left\{s^{1}, \cdots, s^{p+1}\right\}$. Choose a point $r \in S \backslash V(S)$, which is uniquely represented as

$$
r=\sum_{i=1}^{p+1} \beta_{i} s^{i}, \beta_{i} \geq 0 \quad(i=1, \ldots, p+1), \quad \sum_{i=1}^{p+1} \beta_{i}=1 .
$$

For each $i$ such that $\beta_{i}>0$ form the simplex $S_{i}$ defined by $V(S)$ with $s^{i}$ replaced by $r$, i.e., $S_{i}=\operatorname{co}\left\{s^{1}, \cdots, s^{i-1}, r, s^{i+1}, \cdots, s^{p+1}\right\}$, where $\operatorname{co} A$ denotes the convex hull of a set $A$. This division is called radial simplicial division.

The division of $S$ into two subsimplices by choosing the midpoint of one of the longest edges of $S$ as $r$ is called simplicial bisection.

4.4. Rectangular Division. For the case where $W$ is a rectangle defined by (4.3), we propose to use the following rectangular division. Let $S$ be any rectangle of the form $S=\left\{w \in \mathbb{R}_{+}^{p}: \underline{s} \leq w \leq\right.$ $\bar{s}$, where $0 \leq \underline{s} \leq \bar{s}$. Choose a point $r \in S \backslash V(S)$ and an index $j$, and define two vectors $\underline{s}^{\prime}$ and $\bar{s}^{\prime}$ by

$$
\underline{s}^{\prime}=\left(\underline{s}_{1}, \cdots, \underline{s}_{j-1}, r_{j}, \underline{s}_{j+1}, \cdots, \underline{s}_{p}\right) \text { and } \bar{s}^{\prime}=\left(\bar{s}_{1}, \cdots, \bar{s}_{j-1}, r_{j}, \bar{s}_{j+1}, \cdots, \bar{s}_{p}\right) .
$$

Then $S$ is divided into

$$
S_{1}=\left\{w \in \mathbb{R}_{+}^{p}: \underline{s} \leq w \leq \bar{s}^{\prime}\right\} \text { and } S_{2}=\left\{w \in \mathbb{R}_{+}^{p}: \underline{s}^{\prime} \leq w \leq \bar{s}\right\} .
$$

If $r$ is the midpoint of one of the longest edges, the division is called rectangular bisection.

4.5. Exhaustiveness. We recall the exhaustiveness of the division process introduced for the establishment of convergence properties of branch and bound algorithms in global optimization [11].

An infinite nested sequence of subsets $\left\{S^{q}\right\}_{q=1,2, \ldots}$ is said to be exhaustive if $\bigcap_{q=1}^{\infty} S^{q}$ is a singleton. A division process is called exhaustive if each nested infinite subsequence of subsets generated by the process is exhaustive. It is known and easily seen that the simplicial bisection process as well as the rectangular bisection process is exhaustive.

\subsection{Convergence of (GOA).}

Theorem 4.3. Assume that Problem $(P)$ has an optimal solution and that $(G O A)$ with an exhaustive division process generates an infinite sequence $\left\{\left(z^{k}, \lambda^{k}, w^{k}\right)\right\}_{k=1,2, \ldots}$. Then every accumulation point of the sequence is a global optimal solution of Problem $(P)$. 
Proof. We prove the theorem when the simplicial division process and the relaxation problem $\left(\mathrm{P}_{1}(\mathrm{~S})\right)$ are used. The convergence for other cases can be seen in the similar way.

Let $\left(z^{*}, \lambda^{*}, w^{*}\right)$ be an arbitrary accumulation point of $\left\{\left(z^{k}, \lambda^{k}, w^{k}\right)\right\}_{k=1,2, \ldots}$, and let $\left\{z^{\kappa}, \lambda^{\kappa}, w^{\kappa}\right\}_{\kappa \in K}$ be a subsequence converging to $\left(z^{*}, \lambda^{*}, w^{*}\right)$. Take a subsequence if necessary, we can assume that $\lambda^{\kappa i} \underset{\kappa \in K}{\longrightarrow} \lambda^{i *}$ for each $i=1, \ldots, p+1$. From Lemma $3.2\left\{\mu^{\kappa}\right\}_{\kappa \in K}=\left\{\mu\left(S^{\kappa}\right)\right\}_{\kappa \in K}$ is nondecreasing and bounded from above by the optimal value $f^{*}$ of Problem (P). Then there exists a limit $\mu^{*}$ of $\left\{\mu^{\kappa}\right\}_{\kappa \in K}$. By taking further a subsequence if necessary, we can assume that the corresponding sequence $\left\{S^{\kappa}\right\}_{\kappa \in K}$ is nested, i.e., $S^{\kappa+1} \subset S^{\kappa}$ for all $\kappa \in K$.

Since the sets $Z, W, \Omega$ and $\{(z, w): z \in Z, w \in W, B z+w+b=0$,$\} are all closed, it follows$ that

$$
z^{*} \in Z, w^{*} \in W,\left(z^{*}, \lambda^{*}\right) \in \Omega, B z^{*}+w^{*}+b=0 .
$$

Since the division process is exhaustive, it follows that

$$
\bigcap_{\kappa \in K} S^{\kappa}=\left\{s^{*}\right\},
$$

with $s^{*} \in W$, and hence, denoting by $s^{\kappa i}(i=1, \ldots, p+1)$ the vertices of $S^{\kappa}$ we have $s^{\kappa i} \underset{\kappa \in K}{\longrightarrow} s^{*}$ for $i=1, \cdots, p+1$, implying $w^{*}=s^{*}$.

Notice that $\sum_{i=1}^{p+1}\left(s^{\kappa i}\right)^{\top} \lambda^{\kappa i}=0$. Then we obtain

$$
\begin{aligned}
0 & =\lim _{\kappa \in K} \sum_{i=1}^{p+1}\left(s^{\kappa i}\right)^{\top} \lambda^{\kappa i}=\sum_{i=1}^{p+1} \lim _{\kappa \in K}\left(\left(s^{\kappa i}\right)^{\top} \lambda^{\kappa i}\right)=\sum_{i=1}^{p+1}\left(\lim _{\kappa \in K} s^{\kappa i}\right)^{\top}\left(\lim _{\kappa \in K} \lambda^{\kappa i}\right) \\
& =\sum_{i=1}^{p+1}\left(s^{*}\right)^{\top}\left(\lim _{\kappa \in K} \lambda^{\kappa i}\right)=\left(s^{*}\right)^{\top} \sum_{i=1}^{p+1}\left(\lim _{\kappa \in K} \lambda^{\kappa i}\right)=\left(s^{*}\right)^{\top}\left(\lim _{\kappa \in K} \sum_{i=1}^{p+1} \lambda^{\kappa i}\right) \\
& =\left(s^{*}\right)^{\top}\left(\lim _{\kappa \in K} \lambda^{\kappa}\right)=\left(s^{*}\right)^{\top} \lambda^{*} .
\end{aligned}
$$

From (4.4) and (4.5), it follows that $\left(z^{*}, \lambda^{*}, w^{*}\right)$ is a feasible solution of Problem (P). Noting that $\mu^{\kappa} \leq f^{*}$ implies that $\mu^{*} \leq f^{*}$, we see that $\left(z^{*}, \lambda^{*}, w^{*}\right)$ is an optimal solution of $(\mathrm{P})$.

Remark 4.4. In [6], the authors discuss a number of assumptions under which we can obtain the uniformly boundedness of generated sequences. Among others, a technical but quite general assumption is the sequentially bounded constraint qualification (SBCQ). For the problem (PC), this assumption can be represented as follows:

For any convergent sequence $\left\{\left(z^{k}, \lambda^{k}\right): z^{k} \in Z,\left(z^{k}, \lambda^{k}\right) \in \Omega, \lambda^{k} \geq 0\right\}$, there exists for each $k$ a $w^{k}=-B z^{k}-b \geq 0$ satisfying $\left(w^{k}\right)^{\top} \lambda^{k}=0$ and $\left\{\lambda^{k}\right\}$ is bounded.

Since the boundedness of $\left\{\left(z^{k}, \lambda^{k}\right)\right\}$ implies the boundedness of $\left\{w^{k}=-B z^{k}-b\right\}$, a sufficient condition of the $S B C Q$ for the problem $(P C)$ is given by

For any $(z, \lambda)$ satisfying $z \in Z,(z, \lambda) \in \Omega$ and $\lambda \geq 0$, there exists $a w=-B z-b \geq 0$ such that $(w)^{\top} \lambda=0$.

From [7] (Theorem 2, Part (b)), it follows that under some usual constraint qualifications, there exists a constant $c>0$ such that in Problem (PC), constraint $\lambda \geq 0$ can be replaced by the constraint $\|\lambda\| \leq c, \lambda \geq 0$, where $\|\cdot\|$ is any polyhedral norm on $\mathbb{R}^{p}$. Therefore, if we add to the relaxation problem $\left(P_{1}(S)\right)$ the constraints $e^{\top} \lambda^{i} \leq c(i=1, \cdots, \pi)$ and to Problem $\left(P_{2}(S)\right)$ the constraint 
$e^{\top} \lambda \leq c$, where $e=(1, \cdots, 1) \in \mathbb{R}^{p}$, then the existence of an accumulation point of the sequence $\left\{\left(z^{k}, \lambda^{k}, w^{k}\right)\right\}_{k=1,2, \ldots}$ is guaranteed. In particular, for the case that the set

$$
Y=\{(z, \lambda): z \in Z,(z, \lambda) \in \Omega, B z+b \leq 0, \lambda \geq 0\}
$$

is a polyhedron, consider the function

$$
\phi(z, \lambda)=\sum_{i=1}^{p} \min \left\{\lambda_{i},(-B z-b)_{i}\right\}
$$

Then, clearly, the constraint $\lambda^{\top}(B z+b)=0$ is equivalent to the constraint $\phi(z, \lambda)=0$. Since $\phi(z, \lambda)$ is concave, nonnegative over the set $Y$, and takes its minimum over $Y$ at some vertex of $Y$, the constant $c$ can be estimated by the input data defining the polyhedron $Y$.

From a numerical point of view, the algorithm should terminate whenever some approximation of an optimal solution of Problem (P) is found. In the rest of this section, we introduce a concept of approximate optimal solutions of Problem (P), and give a modification of the (GOA) so that it terminates within a finite number of iterations and provides an approximate optimal solution.

Definition 4.5. Given a real numbers $\varepsilon>0$, a point $(z, \lambda, w)$ is called an $\varepsilon$-optimal solution of Problem $(P)$ if it satisfies the following conditions:

(a) $(z, \lambda, w)$ is a feasible solution of Problem $(P)$ with constraint $\lambda^{\top} w=0$ replaced by $\lambda^{\top} w \leq \varepsilon$, and

(b) $f(z, \lambda)$ is a lower bound of the optimal value of Problem (P).

The modification is an additional stopping criterion in Step (i) at each iteration $k$ :

(i)' If $\gamma^{k}=\mu^{k}$, then stop. $\left(\bar{z}^{k}, \bar{\lambda}^{k}, \bar{w}^{k}\right)$ is an optimal solution of Problem (P) with the optimal value $\gamma^{k}$. If $\left(\lambda^{k}\right)^{\top} w^{k} \leq \varepsilon$ for a given $\varepsilon>0$, then stop. $\left(z^{k}, \lambda^{k}, w^{k}\right)$ is an $\varepsilon$-optimal solution of Problem (P).

From Proposition 4.1 and Remark 4.1, we obtain immediately the following result.

Theorem 4.6. Assume that Problem (P) has an optimal solution and that within (GOA) the division process is exhaustive, and the modified step (i)' above is used at each iteration $k$. Then (GOA) always terminates after finitely many iterations yielding an $\varepsilon$-optimal solution of Problem $(P)$.

\section{Illustrative Example and Computational Experiments}

To illustrate the algorithm we present a numerical example which arises from the following linear bilevel programming problem:

$$
\begin{array}{ll}
\min & x_{1}+2 x_{2}+2 y_{1}-y_{2} \\
\text { s.t. } & x_{1}+x_{2}+0.5 y_{1}+y_{2} \leq 6 \\
& x \geq 0, y \geq 0 \\
& y \in \arg \min y_{1}-2 y_{2} \\
& \text { s.t. }-x_{1}+2 x_{2}+y_{2} \leq 4 \\
& -x_{1}-x_{2}+y_{1}+y_{2} \leq 5 \\
& y \geq 0,
\end{array}
$$

where $\arg \min \{q(z): z \in F\}$ denotes the set of optimal solutions of the problem $\min \{q(z): z \in F\}$. 
By setting $z=(x, y)$, and

$$
\begin{gathered}
c=\left(\begin{array}{rrrr}
1.0, & 2.0, & 2.0, & -1.0
\end{array}\right), \\
D=\left(\begin{array}{rrrr}
1.0 & 1.0 & 0.5 & 1.0 \\
-1.0 & 2.0 & 0.0 & 1.0 \\
-1.0 & -1.0 & 1.0 & 1.0
\end{array}\right), \quad d=\left(\begin{array}{r}
-6.0 \\
-4.0 \\
-5.0
\end{array}\right), \\
C=\left(\begin{array}{rrrr}
0.0 & 1.0 & -1.0 & 0.0 \\
1.0 & 1.0 & 0.0 & -1.0
\end{array}\right), \quad q=\left(\begin{array}{r}
1.0 \\
-2.0
\end{array}\right), \\
B=\left(\begin{array}{rrrr}
-1.0 & 2.0 & 0.0 & 1.0 \\
-1.0 & -1.0 & 1.0 & 1.0 \\
0.0 & 0.0 & -1.0 & 0.0 \\
0.0 & 0.0 & 0.0 & -1.0
\end{array}\right), \quad b=\left(\begin{array}{r}
-4.0 \\
-5.0 \\
0.0 \\
0.0
\end{array}\right),
\end{gathered}
$$

Problem (5.1) is transformed to the following program with linear complementarity constraints:

$$
\begin{array}{ll}
\min & c z \\
\text { s.t. } & D z+d \leq 0 \\
& C \lambda+q=0 \\
& B z+b \leq 0 \\
& \lambda^{\top}(B z+b)=0 \\
& z \geq 0, \lambda \geq 0,
\end{array}
$$

which is equivalent to

$$
\begin{array}{ll}
\min & c z \\
\text { s.t. } & D z+d \leq 0 \\
& C \lambda+q=0 \\
& B z+w+b=0 \\
& \lambda^{\top} w=0 \\
& z \geq 0, \quad \lambda \geq 0, w \in W \subset \mathbb{R}_{+}^{4},
\end{array}
$$

where $W$ is a simplex constructed by (4.1)-(4.2).

The vertices of $W$ are $w^{i}=21 e^{i}$ for $i=1, \cdots, 4$, where $e^{i}$ is the $i$ th unit vector of $\mathbb{R}^{4}$, and $w^{5}=0$.

Initialization. Set $S^{1}=W$. Solving the relaxed problem $P_{1}(S)$ we obtain $\left(z\left(S^{1}\right), \lambda\left(S^{1}\right), w\left(S^{1}\right)\right)$ with

$$
z\left(S^{1}\right)=(0.0,0.0,0.0,4.0), \lambda\left(S^{1}\right)=(0.0,2.0,3.0,0.0), w\left(S^{1}\right)=(0.0,1.0,0.0,4.0),
$$

and the optimal value $\mu^{1}=\mu\left(S^{1}\right)=c z\left(S^{1}\right)=-4.0 .\left(z\left(S^{1}\right), \lambda\left(S^{1}\right), w\left(S^{1}\right)\right)$ is not a feasible solution of Problem (5.3). 
Iteration 1. $S^{1}$ is divided into $S_{1}^{1}$ and $S_{2}^{1}$ by a simplicial bisection, where

$$
\begin{gathered}
S_{1}^{1}=\left[\left(\begin{array}{r}
21.0 \\
0.0 \\
0.0 \\
0.0
\end{array}\right),\left(\begin{array}{r}
0.0 \\
21.0 \\
0.0 \\
0.0
\end{array}\right),\left(\begin{array}{r}
0.0 \\
0.0 \\
10.5 \\
10.5
\end{array}\right),\left(\begin{array}{r}
0.0 \\
0.0 \\
0.0 \\
21.0
\end{array}\right),\left(\begin{array}{l}
0.0 \\
0.0 \\
0.0 \\
0.0
\end{array}\right)\right], \\
S_{2}^{1}=\left[\left(\begin{array}{r}
21.0 \\
0.0 \\
0.0 \\
0.0
\end{array}\right),\left(\begin{array}{r}
0.0 \\
21.0 \\
0.0 \\
0.0
\end{array}\right),\left(\begin{array}{r}
0.0 \\
0.0 \\
21.0 \\
0.0
\end{array}\right),\left(\begin{array}{r}
0.0 \\
0.0 \\
10.5 \\
10.5
\end{array}\right),\left(\begin{array}{l}
0.0 \\
0.0 \\
0.0 \\
0.0
\end{array}\right)\right] .
\end{gathered}
$$

Solving Problems $\left.P_{1}\left(S_{1}^{1}\right)\right)$ and $\left.P_{1}\left(S_{2}^{1}\right)\right)$, we respectively obtain

$$
\begin{aligned}
& z\left(S_{1}^{1}\right)=(0.0,0.0,0.0,4.0), \lambda\left(S_{1}^{1}\right)=(0.0,2.0,3.0,0.0), w\left(S_{1}^{1}\right)=(0.0,1.0,0.0,4.0), \\
& \mu\left(S_{1}^{1}\right)=-4.0, \\
& z\left(S_{2}^{1}\right)=(0.0,0.0,0.0,0.0), \lambda\left(S_{2}^{1}\right)=(0.0,2.0,3.0,0.0), w\left(S_{2}^{1}\right)=(4.0,5.0,0.0,0.0), \\
& \mu\left(S_{2}^{1}\right)=0.0 .
\end{aligned}
$$

Thus, $\mu^{2}=-4.0$. No feasible point was found.

At Iteration 11, while considering the simplex

$$
S_{1}^{11}=\left[\left(\begin{array}{l}
2.625 \\
2.625 \\
0.000 \\
5.250
\end{array}\right),\left(\begin{array}{l}
0.000 \\
5.250 \\
0.000 \\
5.250
\end{array}\right),\left(\begin{array}{l}
0.000 \\
0.000 \\
2.625 \\
2.625
\end{array}\right),\left(\begin{array}{l}
0.000 \\
0.000 \\
0.000 \\
5.250
\end{array}\right),\left(\begin{array}{l}
0.000 \\
2.625 \\
0.000 \\
2.625
\end{array}\right)\right],
$$

we obtain

$$
\begin{aligned}
& z\left(S_{1}^{11}\right)=(0.25,0.0,0.0,4.25), \lambda\left(S_{1}^{11}\right)=(2.0,0.0,1.0,0.0), w\left(S_{1}^{11}\right)=(0.0,1.0,0.0,4.25), \\
& \mu\left(S_{1}^{11}\right)=-4.0 .
\end{aligned}
$$

Since $\lambda\left(S_{1}^{11}\right)^{\top} w\left(S_{1}^{11}\right)=0,\left(z\left(S_{1}^{11}\right), \lambda\left(S_{1}^{11}\right), w\left(S_{1}^{11}\right)\right.$ is a feasible solution, and hence an optimal solution with the optimal value $c z\left(S_{1}^{11}\right)=-4.0$.

To test the algorithm, problems of the form (5.2)-(5.3) are chosen. Denote the dimensions of the matrices $D$ and $C$ by $m \times n$ and $n_{1} \times p$, respectively and assume $n_{1}<n$. Accordingly, $d \in \mathbb{R}^{n}$ and $q \in \mathbb{R}^{n_{1}}$. Each element of $D, C, c, d, q$ is randomly generated by using a pseudo-random number from a uniform distribution on $[-1,+1]$.

For each triple $(m, p, n)$ arbitrarily chosen by the programmer, the number $n_{1}$ is set to $\lfloor n / 2\rfloor$. The algorithm was run on 10 thus randomly generated test problems. Typical results (in the sense of average) are summarized in Table 1.

Throughout the algorithm, the simplicial bisection given in Section 4 is used. Moreover, whenever an upper bound $\gamma^{k}$ is found, the stopping criterion $\gamma^{k}=\mu^{k}$ is replaced by $\gamma^{k}-\mu^{k} \leq \delta\left|\gamma^{k}\right|$. For all test problems we chose $\delta=10^{-3}$. It is worth noting that for all test problems, the algorithm terminated by this criterion.

The test was run on a PC 800 MHz Processor, 128 MB RAM, using a FORTRAN 90 code. 


\section{ACKNOWLEDGEMENT}

The main part of this article was written during the first author's stay as visiting professor at Institute of Policy and Planning Sciences, University of Tsukuba, Japan. The authors thank P.B. Hermanns, Department of Mathematics, University of Trier, for carrying out the numerical test reported in Section 5. The second and the third authors are supported by the Grant-in-Aid for Scientific Research B(2) 14380188 and C(2) 13650061 of the Ministry of Education, Culture, Sports, Science and Technology of Japan.

TABLE 1. Computational Results

\begin{tabular}{rrrrrrr}
\hline$m_{1}$ & $m_{2}$ & $n$ & ITER & MAX & FEAS & TIME \\
\hline 5 & 3 & 40 & 28 & 5 & 1 & 1.50 \\
5 & 3 & 60 & 118 & 27 & 2 & 12.36 \\
9 & 3 & 20 & 192 & 6 & 2 & 3.34 \\
9 & 3 & 40 & 143 & 6 & 2 & 8.64 \\
9 & 3 & 80 & 123 & 7 & 1 & 27.13 \\
15 & 3 & 20 & 165 & 5 & 2 & 3.82 \\
15 & 3 & 40 & 315 & 45 & 1 & 23.81 \\
15 & 3 & 60 & 205 & 10 & 2 & 34.68 \\
15 & 3 & 80 & 226 & 12 & 2 & 37.68 \\
\hline 5 & 5 & 20 & 3606 & 226 & 4 & 143.78 \\
5 & 5 & 60 & 4001 & 155 & 4 & 1191.84 \\
5 & 5 & 80 & 7224 & 516 & 4 & 1280.89 \\
9 & 5 & 20 & 11930 & 1143 & 4 & 585.21 \\
9 & 5 & 60 & 5540 & 308 & 2 & 1254.01 \\
9 & 5 & 80 & 19598 & 601 & 3 & 7875.78 \\
15 & 5 & 20 & 2330 & 364 & 5 & 152.26 \\
15 & 5 & 40 & 9571 & 1009 & 3 & 2029.82 \\
15 & 5 & 60 & 8375 & 276 & 4 & 2201.94 \\
\hline 5 & 7 & 20 & 2524 & 214 & 2 & 201.72 \\
5 & 7 & 40 & 21049 & 408 & 5 & 3222.32 \\
5 & 7 & 80 & 10271 & 494 & 5 & 4423.18 \\
9 & 7 & 20 & 18269 & 490 & 1 & 1428.95 \\
9 & 7 & 60 & 91 & 25 & 1 & 35.80 \\
9 & 7 & 80 & 243 & 23 & 2 & 130.08 \\
15 & 7 & 20 & 7028 & 98 & 1 & 763.96 \\
15 & 7 & 60 & 10867 & 301 & 2 & 4656.37 \\
15 & 7 & 80 & 8296 & 446 & 3 & 5866.96 \\
\hline & & & & & &
\end{tabular}

ITER $=$ number of iterations;

MAX = maximal number of subsimplices stored at an iteration;

$\mathrm{FEAS}=$ number of feasible solutions found throughout the algorithm;

TIME $=$ CPU time in seconds. 


\section{REFERENCES}

[1] R.W. Cottle, J.S. Pang, and R.E. Stone: The Linear Complementarity Problem, Academic Press, Boston, 1992.

[2] M. Fukushima, Z.Q. Luo, and J.S. Pang: A Global Convergent Sequential Quadratic Programming Algorithm for Mathematical Programs with Linear Complementarity Constraints, Computational Optimization and Applications Vol. 10, pp. 5-34, 1998.

[3] J. Gotoh, N.V. Thoai, and Y. Yamamoto: Global Optimization Method for Solving the Minimum Maximal Flow Problem, Discussion Paper No. 928, Institute of Policy and Planning Sciences, University of Tsukuba, Japan, 2001.

[4] R. Horst, P. Pardalos, and N.V. Thoai: Introduction to Global Optimization, 2nd Edition, Kluwer Academic Publisher, Dordrecht, 2000.

[5] M. Kojima, N. Megiddo, T. Noma, and A. Yoshise: A Unified Approach to Interior Point Algorithms for Linear Complementarity Problems, Springer-Verlag, Berlin, Heidelberg, 1991.

[6] Z.Q. Luo, J.S. Pang, and D. Ralf: Mathematical Programs with Equilibrium Constraints, Cambridge University Press, 1996.

[7] Z.Q. Luo, J.S. Pang, D. Ralf, and S.Q. Wu: Exact Penalization and Stationarity Conditions of Mathematical Programs with Equilibrium Constraints, Mathematical Programming Vol. 75, pp. 19-76, 1996.

[8] K. Shimizu, Y. Ishizuka, and J.F. Bard: Nondifferentiable and Two-Level Mathematical Programming, Kluwer Academic Publishers, Boston, 1997.

[9] N.V. Thoai: On canonical d.c. programs and applications. In "Essays on Nonlinear Analysis and Optimization Problems". National Center for Scientific Research, pp. 88-100, 1987.

[10] N.V.Thoai: On a class of global optimization problems, Methods of O.R. 58, Eds. P. Kleinschmidt, F.J. Radermacher, W. Schweitzer, H. Wildemann, pp. 115-130, 1989.

[11] N.V. Thoai and H. Tuy: Convergent algorithms for minimizing a concave function. Mathematics of Operations Research Vol. 5, pp. 556-566, 1980.

[12] J.Z. Zhang and G.S. Liu A New Extreme Point Algorithm and Its Application in PSQP Algorithms for Solving Mathematical Programs with Linear Complementarity Constraints, Journal of Global Optimization Vol. 19, pp. 345-361, 2001.

Department of Mathematics, University of Trier, 54286 Trier, Germany; Institute of Policy and Planning Sciences, University of Tsukuba, Tsukuba 305-8573, Japan

E-mail address: thoai@orsun6.uni-trier.de, \{yamamoto, yoshise\}@sk.tsukuba.ac.jp 